\title{
INTELIGENCIA ARTIFICIAL E INNOVACIÓN PARA OPTIMIZAR EL PROCESO DE DIAGNÓSTICO DE LA TUBERCULOSIS
}

\author{
Walter H. Curioso (id), Maria J. Brunette ${ }^{10}{ }^{2, b}$ \\ 1 Universidad Continental, Lima, Perú. \\ ${ }^{2}$ School of Health and Rehabilitation Sciences, The Ohio State University, Ohio, Estados Unidos. \\ a Médico cirujano, doctor en Informática Biomédica; ${ }^{\text {b }}$ ingeniera industrial, doctora en Ingeniería Industrial y de Sistemas.
}

\section{RESUMEN}

La tuberculosis sigue siendo un tema urgente en la agenda de la salud urbana, especialmente en países de medianos y bajos ingresos. Existe la necesidad de desarrollar e implementar soluciones innovadoras y efectivas en el proceso de diagnóstico de la tuberculosis. En este artículo, se describe la importancia de la inteligencia artificial como una estrategia para enfrentar la tuberculosis, mediante un diagnóstico oportuno. Además de los factores tecnológicos, se enfatiza el rol de los factores sociotécnicos, culturales y organizacionales. Se presenta como caso la herramienta eRx que involucra algoritmos de aprendizaje profundo y, en específico, el uso de redes neuronales convolucionales. $\mathrm{eRx}$ es una herramienta prometedora basada en inteligencia artificial para el diagnóstico de tuberculosis que comprende una variedad de técnicas innovadoras que implican el análisis remoto de rayos X para casos sospechosos de tuberculosis. Las innovaciones basadas en herramientas de inteligencia artificial pueden optimizar el proceso de diagnóstico de la tuberculosis y de otras enfermedades transmisibles.

Palabras clave: Tuberculosis; Diagnóstico; Inteligencia Artificial; Invenciones; Salud Urbana; Perú (fuente: DeCS BIREME).

\section{ARTIFICIAL INTELLIGENCE AND INNOVATION TO OPTIMIZE THE TUBERCULOSIS DIAGNOSTIC PROCESS}

\begin{abstract}
Tuberculosis remains an urgent issue on the urban health agenda, especially in low- and middle-income countries. There is a need to develop and implement innovative and effective solutions in the tuberculosis diagnostic process. In this article, We describe the importance of artificial intelligence as a strategy to address tuberculosis control, particularly by providing timely diagnosis. Besides technological factors, the role of socio-technical, cultural and organizational factors is emphasized. The eRx tool involving deep learning algorithms and specifically the use of convolutional neural networks is presented as a case study. $\mathrm{eRx}$ is a promising artificial intelligence-based tool for the diagnosis of tuberculosis; which comprises a variety of innovative techniques involving remote $\mathrm{X}$-ray analysis for suspected tuberculosis cases. Innovations based on artificial intelligence tools can optimize the diagnostic process for tuberculosis and other communicable diseases.
\end{abstract}

Keywords: Tuberculosis; Diagnosis; Artificial Intelligence; Inventions; Urban Health; Peru (source: MeSH NLM).

Citar como: Curioso WH, Brunette MJ. Inteligencia artificial e innovación para optimizar el proceso de diagnóstico de la tuberculosis. Rev Peru Med Exp Salud Publica. 2020;37(3):554-8. doi: https://doi. org/10.17843/rpmesp.2020.373.5585

Correspondencia: Walter H. Curioso; Universidad Continental; Calle Junín 355, Miraflores, Lima, Perú; wcurioso@continental.edu.pe

Recibido: $16 / 04 / 2020$ Aprobado: 08/07/2020 En línea: 20/08/2020

\section{INTRODUCCIÓN}

La salud urbana es una rama de la salud pública que nos permite reflexionar sobre el «impacto en la salud de las intervenciones del sector público en las ciudades, incluyendo aquellas que no necesariamente tienen origen en el sector salud» ${ }^{(1)}$. La salud urbana exhibe una mayor vulnerabilidad a medida que la población mundial se está volviendo más urbana, pero no necesariamente ecológicamente sostenible ${ }^{(2)}$. Vivir en áreas urbanas a menudo implica una mayor exposición a la contaminación del aire libre ${ }^{(3)}$, aumento de temperaturas, falta de acceso a agua potable y desagüe, hacinamiento ${ }^{(4)} \mathrm{y}$ problemas de salud asociados ${ }^{(5)}$, incluso riesgos a contraer enfermedades infecciosas ${ }^{(2)}$.

En ese contexto, la tuberculosis es una enfermedad infecciosa que sigue siendo un desafío en la salud pública de todo el mundo. Según el Informe Mundial de Tuberculosis 2019 de 
la Organización Mundial de la Salud (OMS), alrededor de 1700 millones de personas infectadas con Mycobacterium tuberculosis desarrollarán tuberculosis durante su vida ${ }^{(6)}$. Dicho informe destacó que siete millones de personas con tuberculosis fueron notificadas a los programas nacionales de tuberculosis y reportadas a la OMS ${ }^{(7)}$. Esta situación refleja el escenario complejo que rodea a la carga de tuberculosis en el mundo, especialmente, en países de medianos y bajos ingresos.

Estadísticas recientes demuestran que alrededor del 95\% de las muertes asociadas con la tuberculosis ocurren en países de medianos y bajos ingresos ${ }^{(7)}$. Más allá de la carga de enfermedad por tuberculosis, estos países enfrentan deficiencias en infraestructura, escasez de profesionales de salud, poco entrenados o con experiencia limitada, pacientes que se automedican o consultan solamente a la medicina tradicional y en consecuencia tienen diagnósticos o tratamientos inadecuados ${ }^{(8-10)}$.

Las tasas de tuberculosis tienden a ser mayores en áreas urbanas en comparación con las áreas rurales ${ }^{(2)}$. Así, la tuberculosis sigue siendo un tema relevante en la agenda de la salud urbana y la salud global, particularmente en sistemas de salud débiles de países de medianos y bajos ingresos ${ }^{(11)}$. Otro desafío en los esfuerzos contra la carga de tuberculosis es la alta presencia de pacientes que viven en pobreza o pobreza extrema y que constituyen poblaciones vulnerables, como personas sin hogar, adictos a drogas, migrantes o refugiados ${ }^{(12,13)}$. Por lo tanto, existe la necesidad de desarrollar e implementar soluciones innovadoras y efectivas para atenuar la carga de tuberculosis, específicamente en el proceso de diagnóstico para evitar el incremento de la enfermedad y particularmente, intervenciones que sean oportunas de tal manera que el proceso de diagnóstico sea más eficiente.

El objetivo del artículo es describir la importancia de la inteligencia artificial como una estrategia para enfrentar la tuberculosis, en especial, para proporcionar un diagnóstico oportuno. Se analizan los factores tecnológicos y el rol de los factores sociotécnicos, culturales y organizacionales. Se presenta como caso el proyecto $\mathrm{eRx}$, una herramienta que involucra algoritmos de aprendizaje profundo y el uso de redes neuronales convolucionales que permite detectar anormalidades clínicas preliminares de la tuberculosis.

\section{El rol de la inteligencia artificial y su relación con la salud}

De acuerdo a Chacón Rangel et al., la inteligencia artificial «es la disciplina que busca entender y modelar las capacidades de procesamiento de información de la mente humana, $\mathrm{y}$ comprender los principios generales para explicar y modelar sistemas inteligentes, sean humanos, animales o artificiales» $\mathrm{y}$ «utiliza el computador como medio de simulación» ${ }^{(14)}$.

El campo de la inteligencia artificial ha evolucionado mucho desde 1956, cuando un grupo de investigadores acuñó el térmi- no ${ }^{(15)}$. Si bien ahora hay muchas aplicaciones basadas en inteligencia artificial que se han implementado en países desarrollados, el uso en entornos con limitaciones de recursos sigue siendo relativamente incipiente ${ }^{(15)}$. Siendo los errores de diagnóstico un motivo de seria preocupación en países en vías de desarrollo, las herramientas de inteligencia artificial pueden cumplir un rol importante para optimizar el proceso de diagnóstico de enfermedades infecciosas, incluyendo la tuberculosis y la COVID-19 ${ }^{(16)}$.

No existen clasificaciones universalmente aceptadas de subcampos de inteligencia artificial relevantes para la salud ${ }^{(15)}$. A continuación, se describen de manera suscinta dos de los subcampos de la inteligencia artificial: el aprendizaje computacional y el aprendizaje profundo ${ }^{(16,17)}$. El aprendizaje computacional (machine learning) es un método para automatizar el análisis de datos mediante el uso de algoritmos que identifican iterativamente patrones en los datos y aprenden de ellos ${ }^{(16)}$. El aprendizaje profundo (deep learning) utiliza «diversas cadenas de redes neuronales que además de conformar estructuras complejas secuenciales o en diversas configuraciones, las configuran en roles diferentes» ${ }^{(17)}$. El campo de la inteligencia artificial ha progresado hacia una nueva era de aprendizaje profundo. En particular, las redes neuronales convolucionales (convolutional neural networks) son una clase de aprendizaje profundo que utiliza una red neuronal artificial para analizar imágenes visuales ${ }^{(18)}$. Las redes neuronales convolucionales se han convertido en la técnica preferida para analizar imágenes médicas. Actualmente, se consideran de vanguardia para clasificar imágenes ${ }^{(19)}$.

\section{Comparación de algoritmos de inteligencia artificial usados para el diagnóstico de la tuberculosis}

Hwang et al. fueron los primeros en proponer la implementación de redes neuronales convolucionales para el diagnóstico de tuberculosis ${ }^{(20)}$. Modificaron la arquitectura AlexNet, que utiliza reconocimiento de imágenes generales, según sus preferencias ${ }^{(20)}$. Harris et al. realizaron una revisión sistemática de la precisión diagnóstica de programas basados en inteligencia artificial para identificar anomalías radiológicas (detección asistida por computadora) compatibles con tuberculosis pulmonar en radiografías de tórax ${ }^{(21)}$. Incluyeron 53 estudios, de los cuales 40 fueron de desarrollo y solo 13, clínicos. Los autores concluyeron que los programas de detección asistida por computadora son prometedores, pero la mayoría de estudios se han enfocado en el desarrollo de estos programas más que en la evaluación clínica ${ }^{(21)}$.

\section{Inteligencia artificial para optimizar el diagnóstico de la tuberculosis en el Perú}

En el Perú, los autores (WHC y MJB) forman parte de un equipo interdisciplinario compuesto por investigadores en salud pública, medicina, ingeniería de sistemas de salud y ciencias de la computación que actualmente exploran el uso efectivo de las tecnologías móviles en salud o mSalud 
( $m$ Health) para optimizar las tareas de detección y diagnóstico de la tuberculosis en Lima, Perú ${ }^{(22)}$. En el proyecto, denominado $\mathrm{eRx}$, los profesionales de enfermería o el personal técnico capacitado utilizan teléfonos inteligentes como dispositivos para capturar imágenes de las radiografías de tórax de los pacientes en los establecimientos de salud (Figura 1). Posteriormente, se transmiten dichas imágenes de rayos $\mathrm{X}$ a través de una aplicación (App) previamente instalada dentro del dispositivo móvil (Figura 2) y diseñada en base a métodos de inteligencia artificial utilizando redes neuronales convolucionales. Dicha aplicación permite detectar anormalidades pulmonares y sugiere manifestaciones clínicas preliminares de la tuberculosis ${ }^{(22-25)}$.

Es importante señalar que el proyecto sigue un enfoque de ingeniería de sistemas que se usa ampliamente para mejorar el proceso de atención médica y considera los factores sociotécnicos ${ }^{(26)}$, es decir, analiza las interacciones entre los componentes del sistema para proponer soluciones que integren los factores sociales, técnicos, organizacionales y culturales ${ }^{(26)}$. En esa línea, se debe considerar no solo los factores como la pobre infraestructura física, la escasez del personal de salud, procesos de trabajo redundantes, ineficientes y burocráticos, sino también considerar los factores socioculturales dentro del funcionamiento del sistema de salud, ya que las personas con tuberculosis se encuentran a menudo en situación de pobreza o de pobreza extrema, lo que representa una situación de exclusión social y con limitaciones para el acceso a servicios básicos como salud, educación, vivienda, etc. ${ }^{(26)}$

El proyecto, basado en la aplicación de inteligencia artificial usando tecnologías móviles aplicadas a la salud pública, tiene como objetivo optimizar el proceso de diagnóstico de tuberculosis a través de una herramienta que puede ser utilizada por los profesionales de enfermería o un técnico capacitado en el propio establecimiento de

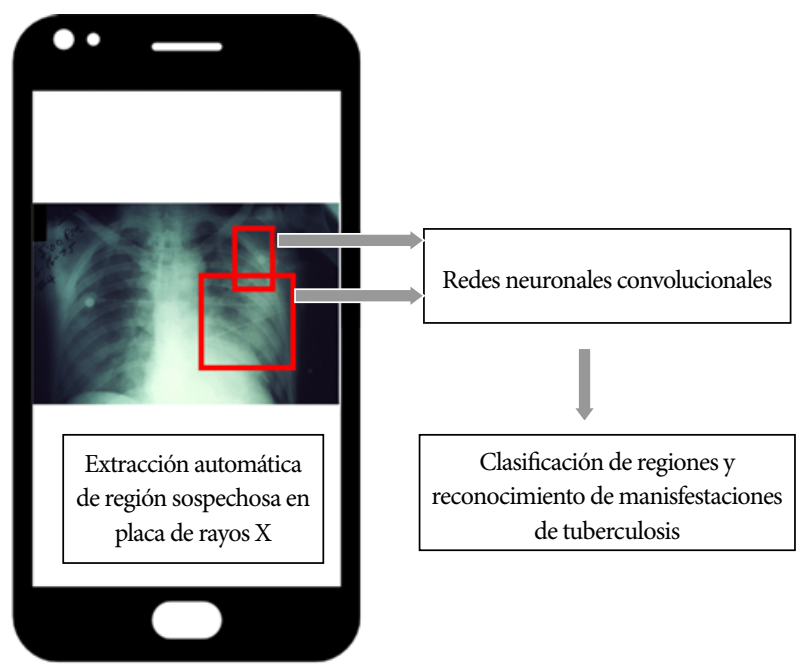

Figura 1. Enfoque propuesto para el análisis de imágenes de rayos X. Imagen de placa de rayos X tomada de http://www.publicdomainfiles. com/show_file.php?id=13519473611917

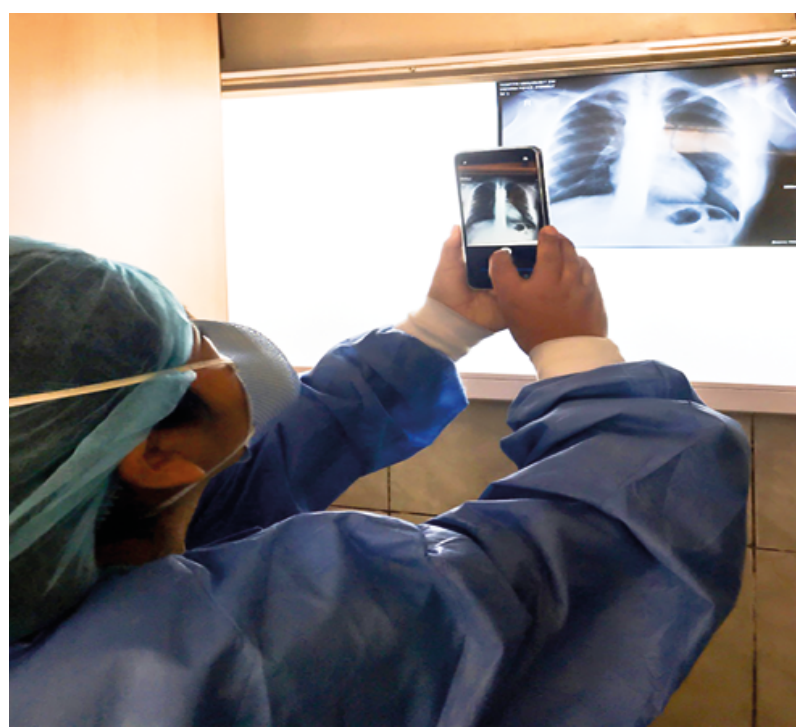

Figura 2. Personal de salud utilizando el aplicativo eRx con un teléfono inteligente en un establecimiento de salud.

salud $^{(22-26)}$. La Figura 3 muestra las interacciones entre el paciente, los profesionales de enfermería o el personal técnico y el servidor del sistema eRx. Durante el periodo comprendido entre septiembre de 2018 y febrero de 2019, se usó el sistema eRx en 5 establecimientos de salud en Huaycán, Ate (Lima). Se contó con la participación de 7 enfermeras y 5 médicos especialistas en neumología. Durante el estudio piloto, se trató de medir las reacciones de los profesionales de salud al interactuar con el sistema eRx, en específico, al utilizar el aplicativo móvil en los establecimientos de salud y a través de la plataforma web en tabletas o computadoras portátiles compactas de pantalla táctil ${ }^{(25)}$. Los profesionales de salud completaron encuestas semanales de retroalimentación. Los resultados de esta evaluación piloto serán reportados en una siguiente publicación.

\section{OBSERVACIONES FINALES}

Las innovaciones basadas en herramientas de inteligencia artificial pueden optimizar el proceso de diagnóstico de la tuberculosis y de muchos otros problemas relacionados con la salud urbana. Así, la flexibilidad de las aplicaciones basadas en la inteligencia artificial permiten superar algunas de las complejidades comunes que rodean la carga de enfermedad por tuberculosis en países de medianos y bajos ingresos, como el poco acceso a establecimientos de salud, las barreras geográficas, el déficit de infraestructura, las elevadas cargas de trabajo del personal de salud, las limitaciones de recursos humanos $y$, en particular, la escasez de profesionales experimentados en técnicas innovadoras para el diagnóstico de tuberculosis.

En específico, el sistema de salud móvil propuesto constituye una herramienta prometedora basada en inteligencia artificial para el diagnóstico de tuberculosis que comprende una variedad 


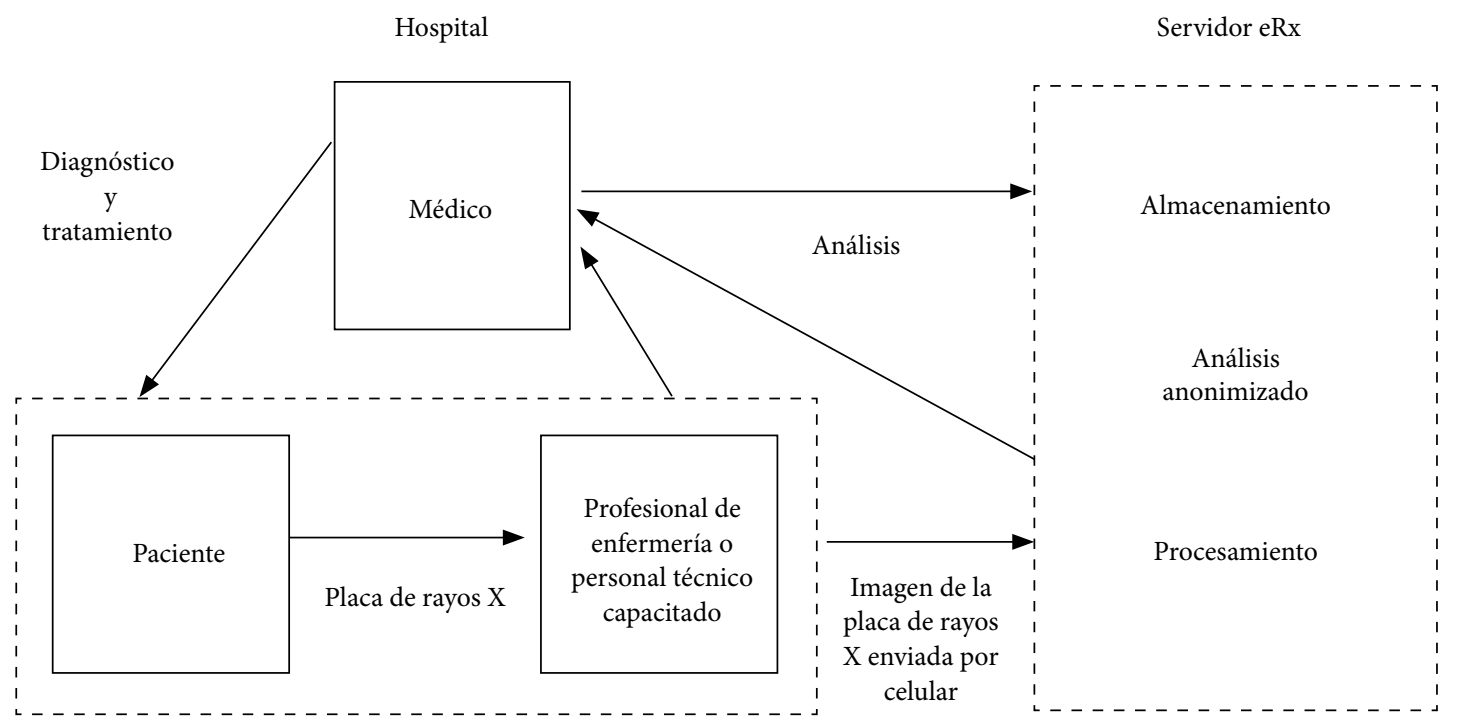

Figura 3. Diagrama que muestra las interacciones entre el paciente, los profesionales de salud y el servidor eRx.

de técnicas innovadoras que implican el análisis remoto de rayos $\mathrm{X}$ para casos sospechosos de tuberculosis. Estas herramientas basadas en inteligencia artificial pueden ser muy útiles en países de medianos y bajos ingresos, sobretodo donde existen serias limitaciones de personal de salud que atiende a pacientes sospechosos de tuberculosis. Incluso, estos sistemas basados en tecnologías móviles pueden implementarse en programas nacionales de telesalud ${ }^{(27)}$.

Existen muchas oportunidades para desarrollar y evaluar algoritmos de diagnóstico asistidos por computadora, como los métodos de aprendizaje computacional que pueden ser integrados en aplicativos para teléfonos móviles con el fin de mejorar la precisión durante las evaluaciones de las radiografías de tórax para identificar casos de tuberculosis pulmonar, e incluso dichos algoritmos pueden ser aplicados en otras enfermedades. En este sentido, la evidencia de las aplicaciones basadas en inteligencia artificial en el proceso de diagnóstico de tuberculosis es muy promisoria, $y$ tiene un gran potencial para futuros usos en la optimización del proceso de diagnóstico de la tuberculosis y de otras enfermedades transmisibles ${ }^{(28)}$.

En esa línea, las oportunidades del telediagnóstico o diagnóstico a distancia aumentan con el crecimiento continuo de la cobertura de Internet y la telefonía móvil, así como, el impulso de proyectos e iniciativas innovadoras relacionadas con la salud móvily la telesalud ${ }^{(27)}$. Esto puede ser de mucha utilidad para el abordaje y optimización de los programas de telediagnóstico y de teleorientación médica, ya que ofrecen muchas posibilidades para superar barreras, como la escasez de infraestructura, la falta de acceso a servicios y de recursos humanos, para brindar atención oportuna a los pacientes, en especial, a los más vul- nerables que viven principalmente en zonas marginadas y de elevada pobreza urbana.

Con el desarrollo actual de las ciudades, se hacen cada vez más necesarias las soluciones basadas en inteligencia artificial. Es muy probable que muchas de las herramientas tecnológicas y acceso a Internet estén cada vez más disponibles y potencialmente, más integradas, en los esfuerzos de diagnóstico rutinario de la tuberculosis, en especial en las zonas más necesitadas y donde se encuentra la población más vulnerable. Sin embargo, se debe considerar también las diferentes características sociodemográficas y el perfil epidemiológico de las distintas poblaciones de pacientes. No obstante, estas soluciones pueden facilitarse con la promoción de redes de colaboración, importantes para compartir algoritmos y buenas prácticas.

Asimismo, se deben compartir bases de datos de imágenes de rayos $\mathrm{X}$ de manera pública y en repositorios interoperables de acceso abierto, ya que actualmente las bases de datos disponibles son limitadas y no adecuadamente anotadas ${ }^{(29)}$. Esto no solo beneficiará a los programas de entrenamiento en radiología o en programas de educación médica continua, sino que permitirá mejorar la precisión de los algoritmos de aplicaciones basados en inteligencia artificial en el mundo.

Finalmente, es imprescindible incluir estos temas en los programas de entrenamiento en informática biomédica, salud digital e ingeniería biomédica ${ }^{(27)}$. Esta es una gran oportunidad de colaborar entre entidades del gobierno central con la academia, desde donde se pueden promover investigaciones relacionadas a la evaluación a escala masiva de estas aplicaciones considerando diseños metodológicos rigurosos ${ }^{(27)}$, y teniendo en cuenta un enfoque centrado en el usuario y la influencia de los factores sociotécnicos. 
Contribuciones de autoría: WHC y MJB han participado en la concepción y diseño del artículo, en la recolección de información bibliográfica, en la redacción del manuscrito y en la revisión crítica del artículo. Todos los autores aprobaron la versión final del artículo y se hacen responsables de los aspectos que integran el manuscrito.
Fuentes de financiamiento: Este trabajo fue financiado parcialmente por el proyecto: «A sociotechnical systems approach to improve tuberculosis diagnostics in Peru» NIH/NIBIB 5R01EB021900-04.

Conflictos de interés: Los autores declaran no tener conflictos de interés en la publicación de este artículo.

\section{REFERENCIAS BIBLIOGRÁFICAS}

1. Caiaffa WT, Friche AADL, Ompad DC. Salud urbana: Hitos, dilemas, perspectivas y desafíos. Cad Saude Publica. 2015;31:S5-S6. doi: 10.1590/0102-311XED01S115.

2. Grover A, Singh RB. Urban Health and Wellbeing. Indian Cases Studies. Singapore:Springer; 2020. doi: 10.1007/978-981-13-6671-0.

3. Lu JG. Air pollution: A systematic review of its psychological, economic, and social effects. Curr Opin Psychol. 2020;32:52区65. doi: 10.1016/j. copsyc.2019.06.024.

4. Anthonj C, Setty KE, Ezbakhe F, Manga M, Hoeser C. A systematic review of water, sanitation and hygiene among Roma communities in Europe: Situation analysis, cultural context, and obstacles to improvement. Int J Hyg Environ Health. 2020;226:113506. doi: 10.1016/j.ijheh.2020.113506.

5. The SALURBAL Team. The Salud Urbana en América Latina (SALURBAL) Project: Learning from Latin America’s Cities for a Healthier Future. En: Gatzweiler F. (eds) Urban Health and Wellbeing Programme. Urban Health and Wellbeing (Systems Approaches). Singapore: Springer; 2020. doi: 10.1007/978-981-15-1380-0.

6. World Health Organization (WHO) [Internet]. Global tuberculosis report; 2019. [citado el 14 de abril de 2020]. Disponible en: https://apps.who. int/iris/bitstream/handle/10665/329368/9789241565714-eng.pdf?ua=1.

7. World Health Organization (WHO) [Internet]. Tuberculosis: key facts; 2020 [citado el 14 de abril del 2020]. Disponible en: https://www.who.int/ en/news-room/fact-sheets/detail/tuberculosis.

8. Furin J, Cox H, Pai M. Tuberculosis. Lancet. 2019;393(10181):1642-1656. doi: 10.1016/S0140-6736(19)30308-3.

9. MacNeil A, Glaziou P, Sismanidis C, Maloney S, Floyd K. Global Epidemiology of Tuberculosis and Progress Toward Achieving Global Targets - 2017. MMWR Morb Mortal Wkly Rep. 2019;68(11):263-266. doi: 10.15585/mmwr.mm6811a3.

10. Alarcón V, Alarcón E, Figueroa C, Mendoza-Ticona A. Tuberculosis in Peru: epidemiological situation, progress and challenges for its control. Rev Peru Med Exp Salud Publica. 2017;34(2):299-310. doi: 10.17843/ rpmesp.2017.342.2384.

11. Noykhovich E, Mookherji S, Roess A. The Risk of Tuberculosis among Populations Living in Slum Settings: a Systematic Review and Meta-analysis. J Urban Health. 2019;96(2):262-275. doi: 10.1007/s11524-018-0319-6.

12. Abubakar I, Matteelli A, de Vries G, Zenner D, Cirillo DM, Lönnroth K, et al. Towards tackling tuberculosis in vulnerable groups in the European Union: the E-DETECT TB consortium. Eur Respir J. 2018;51(5). doi 10.1183/13993003.02604-2017.

13. Gianella C, Pesantes MA, Ugarte-Gil C, Moore DAJ, Lema C. Vulnerable populations and the right to health: lessons from the Peruvian Amazon around tuberculosis control. Int J Equity Health. 2019;18(1):28. doi: 10.1186/s12939-019-0928-Z

14. Chacón JG, Flórez AS, Rodríguez E. La inteligencia artificial y sus contribuciones a la física médica y la bioingeniería. Revista MundoFesc. 2015;1(9):60-63.

15. Wahl B, Cossy-Gantner A, Germann S, Schwalbe NR. Artificial intelligence (AI) and global health: how can AI contribute to health in resource-poor settings?. BMJ Glob Health. 2018;3(4):e000798. doi: 10.1136/bmigh-2018-000798.

16. Rajkomar A, Dean J, Kohane I. Machine Learning in Medicine. N Engl J Med. 2019;380(14):1347-1358. doi: 10.1056/NEJMra1814259.

17. Lee HC, Jung CW. Anesthesia research in the artificial intelligence era. Anesth Pain Med. 2018;13(3):248-255. doi: 10.17085/apm.2018.13.3.248.

18. Meraj SS, Yaakob R, Azman A, Rum SNM, Nazri ASA. Artificial Intelligence in Diagnosing Tuberculosis: A Review. International Journal on Advanced Science, Engineering and Information Technology. 2019;9(1):81-91. doi: 10.18517/ijaseit.9.1.7567.

19. Lakhani P, Sundaram B. Deep learning at chest radiography: automated classification of pulmonary tuberculosis by using convolutional neural networks. Radiology. 2017;284(2): 574-582. doi: 10.1148/radiol.2017162326.

20. Hwang S, Kim H-E., Jeong J, Kim H-J. A novel approach for tuberculosis screening based on deep convolutional neural networks. SPIE Medical Imaging, International Society for Optics and Photonics. 2016;9785:97852W1. doi: 10.1117/12.2216198.

21. Harris M, Qi A, Jeagal L, Torabi N, Menzies D, Korobitsyn A, et al. A systematic review of the diagnostic accuracy of artificial intelligence-based computer programs to analyze chest $\mathrm{x}$-rays for pulmonary tuberculosis. PLoS One. 2019;14(9):e0221339. doi: 10.1371/journal.pone.0221339.

22. Cao Y, Liu C, Zhang N, Liu B, Sun T, Zhang P, et al. Improving Tuberculosis Diagnostics using Deep Learning and Mobile Health Technologies among Resource-poor and Marginalized Communities. 2016 IEEE First Conference on Connected Health: Applications, Systems and Engineering Technologies. 2016. doi: 10.1109/CHASE.2016.18.

23. Alcantara MF, Cao Y, Liu C, Liu B, Brunette M, Zhang N, et al. Improving Tuberculosis Diagnostics using Deep Learning and Mobile Health Technologies among Resource-poor Communities in Perú. Smarth Health. 2017(1-2):66-76. doi: 10.1016/j.smhl.2017.04.003.

24. Liu C, Cao Y, Alcantara M, Liu B, Brunette M, Peinado J, et al. TXCNN: Detecting tuberculosis in chest $\mathrm{X}$-ray images using convolutional neural network. 2017 IEEE International Conference on Image Processing (ICIP). 2017. doi: 10.1109/ICIP.2017.8296695.

25. Alcantara MF, Cao Y, Liu B, Liu C, Zhang N, Zhang P, et al. eRx - A technological advance to speed-up TB diagnostics. Smarth Health. 2020(16):100117. doi: 10.1016/j.smhl.2020.100117.

26. Brunette MJ, Curioso WH. Integrated mobile health systems: Role of socio-cultural factors and the socio-technical systems approach. Rev Peru Med ExpSalud Publica. 2017;34(3):544-550. doi: 10.17843/rpmesp.2017.343.2859.

27. Curioso WH. Building Capacity and Training for Digital Health: Challenges and Opportunities in Latin America. J Med Internet Res. 2019;21(12):e16513. doi: 10.2196/16513.

28. Apostolopoulos ID, Mpesiana TA. Covid-19: automatic detection from $\mathrm{X}$-ray images utilizing transfer learning with convolutional neural networks. Phys Eng Sci Med. 2020. doi: 10.1007/s13246-020-00865-4.

29. Jaeger S, Candemir S, Antani S, Wáng YX, Lu PX, Thoma G. Two public chest X-ray datasets for computer-aided screening of pulmonary diseases. Quant Imaging Med Surg. 2014;4(6):475-7. 